\title{
Estimation of surface-layer scaling parameters in the unstable boundary layer: implications for orographic flow speed-up
}

Article

Accepted Version

Argain, J. L., Teixeira, M. and Miranda, P. M. A. (2017) Estimation of surface-layer scaling parameters in the unstable boundary layer: implications for orographic flow speed-up. Boundary-Layer Meteorology, 165 (1). pp. 145-160. ISSN 0006-8314 doi: https://doi.org/10.1007/s10546-017-0260-3 Available at https://centaur.reading.ac.uk/70672/

It is advisable to refer to the publisher's version if you intend to cite from the work. See Guidance on citing.

Published version at: https://link.springer.com/article/10.1007/s10546-017-0260-3

To link to this article DOI: http://dx.doi.org/10.1007/s10546-017-0260-3

Publisher: Springer

All outputs in CentAUR are protected by Intellectual Property Rights law, including copyright law. Copyright and IPR is retained by the creators or other copyright holders. Terms and conditions for use of this material are defined in the End User Agreement. 


\section{CentAUR}

Central Archive at the University of Reading

Reading's research outputs online 


\section{Estimation of surface-layer scaling parameters in the unstable 2 boundary-layer: implications for orographic flow speed-up}

3

4 José Luís Argaín • Miguel A. C. Teixeira • Pedro M. A. Miranda

5

6

7 Received: DD Month YEAR/ Accepted: DD Month YEAR

8 Abstract A method is proposed for estimating the surface-layer depth $\left(z_{S}\right)$ and the 9 friction velocity $\left(u_{*}\right)$ as a function of stability (here quantified by the Obukhov length, $L$ )

10 over the complete range of unstable flow regimes. This method extends the one

11 developed previously by the authors for stable conditions in Argaín et al. (Boundary-

12 Layer Meteorol, 2009, Vol.130, 15-28), but uses a qualitatively different approach. The

13 method is specifically used to calculate the fractional speed-up $(\Delta S)$ in flow over a ridge,

14 although it is suitable for more general boundary-layer applications. The behaviour of

$15 z_{S}(L)$ and $u_{*}(L)$ as a function of $L$ is indirectly assessed via calculation of $\Delta S(L)$ using

16 the linear model of Hunt et al. (Q J R Meteorol Soc, 1988, Vol.29, 16-26) and its

17 comparison with the field measurements reported in Coppin et al. (Boundary-Layer

18 Meteorol, 1994, Vol.69, 173-199) and with numerical simulations carried out using a

19 nonlinear numerical model, FLEX. The behaviour of $\Delta S$ estimated from the linear model

20 is clearly improved when $u_{*}$ is calculated using the method proposed here, confirming

21 the importance of accounting for the dependences of $z_{S}(L)$ and $u_{*}(L)$ on $L$ to better

22 represent processes in the unstable boundary-layer.

José Luis Argaín

Departamento de Física, FCT, Universidade do Algarve, Faro 8005-139, Portugal. jargain@ualg.pt

Miguel Ângelo Cortez Teixeira Department of Meteorology, University of Reading, Earley Gate, PO Box 243, Reading RG6 6BB, UK m.a.teixeira@reading.ac.uk

Pedro Manuel Alberto de Miranda Instituto Dom Luiz, Faculdade de Ciências, Universidade de Lisboa, Lisbon 1749-016, Portugal pmmiranda@fc.ul.pt 
24 Keywords Convective boundary layer $\cdot$ Flow speed-up $\cdot$ Friction velocity $\cdot$ Surface-layer

25 height $\cdot$ Unstable stratification

\section{$27 \quad 1$ Introduction}

28 Fractional speed-up $(\Delta S)$ of flow over hills or mountains is defined as the ratio of the

29 speed perturbation at a given height to the upstream, unperturbed flow speed at the same

30 height. This quantity is highly relevant both from meteorological and wind engineering

31 perspectives, since it characterizes the modulation of the wind speed by orography. Hunt

32 et al. (1988) (hereafter HLR) developed one of the first theoretical linear atmospheric

33 boundary layer (ABL) models of flow over hills, which is one of the simplest and

34 computationally cheapest tools available for estimating $\Delta S$.

35 However, stratification affects $\Delta S$ and must be carefully accounted for in the evaluation

36 of the scaling parameters that characterize the ABL. Among these, a key parameter is the

37 friction velocity $\left(u_{*}\right)$, and another one is the surface-layer depth $\left(z_{s}\right)$, usually estimated as

$385 \%$ to $10 \%$ of the ABL depth.

39 Weng (1997) (hereafter W97), after implementing a continuous wind profile in the HLR

40 model, found that his predictions of $\Delta S$ disagreed significantly with the observations of

41 Coppin et al. (1994) (hereafter C94). Argaín et al. (2009) (hereafter A09) showed that

42 these discrepancies were due to the fact that the calculations in W97 were carried out

43 assuming that $u_{*}$ is constant, regardless of the different observed stability regimes. They

44 proposed a method for estimating $u_{*}$ in stably-stratified flows, which has led to an

45 improved prediction of $\Delta S$ over 2D hills. C94 also compared their observations with

46 predictions from the HLR model, and found considerable disagreement, both in stable

47 and unstable conditions. Here we show that, as in stably-stratified flows, a decisive

48 reason for such disagreements in unstable flow is the assumption of constant $u_{*}$.

49 In the present study, a new method is developed for estimating $z_{S}$ and $u_{*}$ as a function of

50 stability (here quantified by the Obukhov length, $L$ ) over the complete unstable

51 stratification range, i.e. from the free-convection to the neutral stability limits. Procedures

52 are developed for estimating $z_{s}$ in a neutral $\mathrm{ABL}$, and for estimating this and several

53 other scaling parameters, such as $u_{*}, L$ and Deardorff's convective velocity scale, $w_{*}$, in

54 the free-convection regime, which are preliminary steps for defining $z_{S}(L)$ and $u_{*}(L)$ for 
55 all stabilities. Given that the physical processes taking place in the CBL and in an 56 unstable surface layer are substantially different from those in a stable boundary ABL, 57 the method used to represent them also differs substantially, requiring the use of 58 additional theory.

59 The main motivation for developing this new method for estimating $z_{S}(L)$ and $u_{*}(L)$ is 60 the calculation of $\Delta S(L)$ for unstable flow over hills, although it must be noted that the 61 method can also be used for more general boundary layer applications. The calculation of $62 \Delta S(L)$ requires knowing $u_{*}(L)$ which, in the method proposed here, also requires 63 estimating $z_{S}(L)$. The behaviour of $z_{S}(L)$ and $u_{*}(L)$ is thus indirectly assessed through

64 the calculation of $\Delta S(L)$ using the HLR model. These predictions are compared with field 65 measurements, reported in C94, and numerical simulations, carried out using a 2D 66 microscale-mesoscale non-hydrostatic model, FLEX. These comparisons allow us to 67 show how $z_{S}(L)$ and $u_{*}(L)$ are sometimes not estimated in a physically consistent way, a 68 limitation that the present method aims to overcome.

69 Section 2 presents the method that accounts for unstable stratification in the ABL and its 70 calibration. Section 3 describes the main results, namely comparisons between theory, 71 numerical simulations and measurements, using the new unstable ABL formulation. 72 Finally, Sect. 4 summarizes the main conclusions of this study.

\section{Methodology}

$75 \quad 2.1$ Unstable ABL model

76 Several studies show that the ABL, under moderately to strongly unstable stratification

77 (usually known as CBL), can be represented by a simplified three-layer bulk model (e.g.,

78 Garratt 1992). This comprises a thin statically unstable surface layer of depth $z_{s}$, a well79 mixed layer, of height $z_{i}$ and depth $\Delta z_{i}=z_{i}-z_{S}$, and a transition layer of thickness $80 \Delta z_{c i}$, coinciding with a temperature inversion capping the mixed layer, which inhibits 81 vertical mixing. In the mixed layer, quantities such as the mean potential temperature $(\theta)$ 82 and wind velocity $(U, V)$ are well-mixed, and therefore constant with height, i.e. $\theta(z)=$ 83 const., $U(z)=$ const. and $V(z)=0$. For our purposes, the strict fulfilment of these 84 profile shapes in the mixed layer is not critical, since we are essentially interested in the 85 surface layer, for which typically $z_{s} \approx 0.05 z_{i}$ to $0.1 z_{i}$ (Stull 1988). In the surface layer we 
86 assume that the turbulent shear stresses have much more important effects on the mean

87 flow than the Coriolis force. Hence, the Coriolis parameter $(f)$ is set to zero, except

88 where otherwise explicitly stated. Since the surface layer has characteristics which make

89 it markedly different from the mixed layer, $z_{S}$ can be defined as an important length scale

90 of the ABL, essential for describing the impact of the orography on the wind profile. This

91 follows McNaughton (2004), who established $z_{S}$ as a new basis parameter for similarity

92 models of the surface layer. In the method proposed here, $z_{s}$ is essential for estimating

93 the key velocity scale, $u_{*}$, and hence for calculating $\Delta S(L)$.

94

$95 \quad 2.2$ Surface-layer model

96 According to Monin-Obukhov similarity theory (MOST), in the surface layer the non-

97 dimensional vertical gradients of $U(z)$ and $\theta(z)$ are universal functions of the parameter

$98 z / L$, taking the forms

99

$$
\Phi_{m}\left(\frac{z}{L}\right)=\frac{\kappa z}{u_{*}} \frac{\partial U}{\partial z},
$$

100 and

$$
\Phi_{h}\left(\frac{z}{L}\right)=\frac{\kappa z}{\operatorname{Pr}_{t} \theta_{*}} \frac{\partial \theta}{\partial z},
$$

102 where $z$ is the height above the effective ground level, $\kappa$ is the von Kármán constant, $103 \operatorname{Pr}_{t}$ is the turbulent Prandtl number and $\theta_{*}$ represents the surface-layer scaling 104 temperature. $u_{*}$ and $\theta_{*}$, are defined using the vertical eddy kinematic fluxes of 105 momentum and heat at the surface, i.e. $u_{*}^{2}=\left(\overline{w^{\prime} u^{\prime}}\right)_{0}$ and $\theta_{*}=-\left(\overline{w^{\prime} \theta}\right)_{0} / u_{*}$. The length

$106 L$ is given by

$$
L=-\frac{\theta_{0} u_{*}^{3} / \kappa}{g\left(\overline{w^{\prime} \theta^{\prime}}\right)_{0}}=\frac{\theta_{0} u_{*}^{2}}{g \kappa \theta_{*}},
$$

108 where $\theta_{0}$ is the potential temperature at the surface and $g$ is the gravitational 109 acceleration. Wilson (2001) (hereafter W01), after analyzing several forms of the 110 functions $\Phi_{m}$ and $\Phi_{h}$, proposed the following general form for the unstable regime $(z / L$ $111<0)$, 


$$
\Phi=\left(1+\gamma\left|\frac{Z}{L}\right|^{\alpha_{1}}\right)^{-\alpha_{2}}
$$

113 which is valid for both $\Phi_{m}$ and $\Phi_{h}$. He noted that in order to obtain the correct physical

114 behaviour for the gradients $\partial U / \partial z$ and $\partial \theta / \partial z$ in the free-convection limit $(z / L \rightarrow-\infty)$, it

115 is required that $\alpha_{1} \alpha_{2}=1 / 3$. For this combination of values (4) behaves in this limit

116 similarly to 'classical' free-convection expressions, with $\partial U / \partial z$ and $\partial \theta / \partial z$ varying

117 proportionally to $z^{-4 / 3}$. He further noted that, for this choice of parameters, (1) - (2) may

118 be integrated straightforwardly. Following W01 we will use $\kappa=0.4, P r_{t}=0.95, \gamma_{h}=$

$1197.86, \alpha_{1 m}=\alpha_{1 h}=2 / 3, \alpha_{2 m}=\alpha_{2 h}=1 / 2$ and $\gamma_{m}=3.59$.

120 Subscripted indices $s, n$ and $f c$ hereafter denote values of flow parameters in the surface

121 layer, in the neutral regime $(|L| \rightarrow \infty)$, and in the free-convection regime $(|L| \rightarrow 0)$,

122 respectively.

123 The method developed here requires that $z_{s f c}, u_{* f c}, L_{f c}, z_{s n}, u_{* n}$ and $z_{0}$, be known in

124 order to calculate $u_{*}(L)$ and $z_{S}(L)$. The primary input parameters are $u_{* n}, z_{i f c}$ and the

125 aerodynamic roughness height, $z_{0}$, which must be provided initially.

$127 \quad 2.3$ Estimating parameters in the free-convection and neutral regimes

128 MOST shows good agreement with observations in regimes with sufficiently strong 129 winds (high values of $u_{*}$ ) or under relatively low surface heat flux, $\left(\overline{w^{\prime} \theta}\right)_{0}$, where $|L|>$ $13010^{2} \mathrm{~m}$. This theory is based on the assumption that, in the surface layer, $z$ and $L$ are the 131 only relevant turbulence length scales. While this assumption is valid for relatively small 132 values of $|z / L|$ (say $|z / L|<1$ ), for larger values, in particular in the free-convection 133 regime, MOST becomes incomplete. In the perfectly windless regime, purely dominated 134 by thermal effects, both the mean wind speed and $u_{*}$ approach zero, and MOST produces 135 singularities and underestimates the surface fluxes. However, perfectly windless 136 conditions occur very rarely, and the theory can still be applied, if conjugated with CBL 137 theory, for low but non-zero winds, as will be shown below.

138 For the highly convective ABL, Deardorff (1970) suggested the following convective 139 velocity scale 


$$
w_{*}=\left[\frac{g}{\theta_{0}}\left(\overline{w^{\prime} \theta^{\prime}}\right)_{0} z_{i f c}\right]^{1 / 3} .
$$

141 The combination of MOST and Deardorff similarity theory, adopted here, provides a 142 model that is consistent throughout the whole CBL (Kaimal et al. 1976) (hereafter K76), 143 and for stabilities ranging from the neutral regime to the free-convection regime. This 144 latter regime does not strictly correspond to $L=0$, but rather to a minimum, suitably 145 small value of $L=L_{f c}$, to be determined. In the free-convection regime we need to 146 estimate $z_{s f c}, u_{* f c}$ and $L_{f c}$. Given that $u_{* f c}$ is defined in relation to $w_{*}$ (as shown 147 below), this latter quantity, defined by (5), must also be related to the known input 148 parameters. This requires a total of four equations (see below).

149 Many observations have confirmed that the transition from the shear-driven turbulent 150 regime of the surface layer to the buoyancy driven regime of the mixed layer usually 151 occurs at a height of order $|L|$. Hence, in a highly-convective ABL (Garratt 1992),

$$
z_{s f c}=c_{f c}\left|L_{f c}\right|,
$$

153 where $c_{f c}=2$. Equation 6 will be adopted hereafter in the free-convection regime.

154 Based on observations, Schumann (1988) (hereafter S88) assumed that $z_{s} / z_{i}=0.1$. As

155 will be seen later, this assumption is too restrictive over the whole stability interval,

156 since, $z_{i}$ is expected to increase and $z_{s}$ to decrease as the stratification becomes more

157 unstable. A more general definition of $z_{S}$ is thus required. This is developed in Sect. 2.4.

158 Businger (1973) proposed the idea that $u_{*}$ does not vanish at low wind speeds, 159 introducing the concept of a 'minimum friction velocity', valid in the free-convection 160 regime $\left(u_{* \min }=u_{* f c}\right)$. Combining (3) and (5) in this regime, we obtain

$$
\frac{\kappa\left|L_{f c}\right|}{Z_{i f c}}=\left(\frac{u_{* f c}}{w_{*}}\right)^{3} .
$$

162 Using (6), it can be easily shown from (7) that $z_{s f c} / z_{i f c}$ decreases as $u_{* f c} / w_{*}$ decreases, 163 which is physically plausible.

164 Various authors, such as S88 and Sykes et al. (1993) (hereafter S93), have advocated the 165 view that $u_{* f c} / w_{*}$ is a function of $z_{s f c} / z_{0}$ or $z_{i f c} / z_{0}$ as well. Following the less general 166 relations derived by S88 and S93, valid only for limited intervals of $z_{0}$, Zilitinkevich et 
167 al. (2006) (hereafter Z06) suggested a more complete formulation for the relationship 168 between $u_{* f c} / w_{*}$ and $z_{i f c} / z_{0}$, which takes into account the combined effects of 169 buoyancy and shear forces,

$$
\begin{array}{ll}
\frac{u_{* f c}}{w_{*}}=c_{1}\left[\ln \frac{z_{i f c} / z_{0}}{\left(\ln z_{i f c} / z_{0}-c_{0}\right)^{3}}+c_{2}\right]^{-1} & \text { for } z_{i f c} / z_{0} \geq \sigma, \\
\frac{u_{* f c}}{w_{*}}=c_{3}\left[\frac{z_{0}}{z_{i f c}}+c_{4}\left(\frac{z_{0}}{z_{i f c}}\right)^{8 / 7}\right]^{1 / 6} & \text { for } z_{i f c} / z_{0}<\sigma,
\end{array}
$$

172 where $\sigma=3.45 \times 10^{5}, u_{* f c} / w_{*}(\sigma)=0.065, c_{0}=6.00, c_{1}=0.29, c_{2}=-2.56, c_{3}=0.54$

173 and $c_{4}=0.3$. Equations 8 and 9 agree very well with both LES and field data in the free-

174 convection regime (Z06), and incorporate the best characteristics of the S88 and S93 175 models.

176 The height $z_{i}$ characterizes the PBL in a fairly integrated manner, being closely related to 177 fundamental quantities such as $\left(\overline{w^{\prime} \theta}\right)_{0}$. For this reason, as a first approach, we suggest

178 estimating the surface-layer scaling parameters in the free-convection regime based on a 179 known value of $z_{i f c}$. This allows obtaining $u_{* f c} / w_{*}$ directly from (8) - (9), since $z_{0}$ is 180 also assumed to be known.

181 Our final constraint is based on Venkatram (1978) who, by using a simple mixed-layer 182 model for the CBL, derived the following relationship between $w_{*}$ and $z_{i f c}$,

$$
w_{*}=c_{5} Z_{i f c},
$$

184 where $c_{5}=1.12 \times 10^{-3} \mathrm{~s}^{-1}$. Equation 10 compares extremely well with observations (see 185 Appendix 2). Using the available value of $z_{i f c}$, (10) allows us to determine $w_{*}$ directly.

186 Equations 6 - 10 may thus be used to obtain the surface-layer parameters in the free187 convection regime, as follows. Given $z_{i f c}$ and $z_{0},(8)$ or (9) is used to obtain $u_{* f c} / w_{*}$ 188 and (10) is used to obtain $w_{*}$, which yields $u_{* f c}$. Given $z_{i f c}, w_{*}$ and $u_{* f c}$, determined in 189 the preceding step, (7) is used to obtain $L_{f c}$. Finally, $L_{f c}$ is inserted into (6) to obtain $z_{s f c}$. 190 This yields $u_{* f c}, L_{f c}$ and $z_{s f c}$, as required. Several different procedures analogous to the 191 one just described would be possible, depending on what input parameters are known 192 initially. 
According to MOST, in the neutral regime

$$
U_{n}(z)=\frac{u_{* n}}{\kappa} \ln \left(\frac{z}{z_{0}}\right) .
$$

195 Since, from (6), $z_{S}$ is expected to depend on $L$, in the neutral regime at least (where no

196 stability effects exist), it seems reasonable to assume $z_{s}$ to be a fixed fraction of $z_{i}$ (Stull 197 1988),

$$
Z_{s n}=c_{S L} Z_{i n},
$$

199 where that fraction is conventionally defined as $5 \%$ to $10 \%$ of $z_{i}$ (Stull 1988). In our 200 model, we assume $c_{S L}=0.05$ (following Stull 2011). Here, and unlike what previous 201 authors have done, (12) is adopted only for the strictly neutral regime. As will be seen 202 later (Sect. 3.2), (12) holds approximately for a weakly unstable ABL, but not for a 203 strongly unstable ABL. In order to obtain $z_{s n}$ from (12), it is still necessary to estimate $204 z_{i n}$. This can be done using the expression of Rossby and Montgomery (1935),

$$
Z_{i n}=\frac{C_{\text {zin }} u_{* n}}{|f|},
$$

206 where $c_{\text {zin }}=0.2$ (Garratt 1992).

2082.4 Estimating $z_{s}$ and $u_{*}$ for arbitrary $L<0$

209 The preceding section described the methodologies for estimating all the parameters 210 required for defining $u_{*}$ and $z_{s}$ in the free-convection and neutral regimes. Next we 211 explain the approach used to estimate these two parameters for arbitrary $L<0$.

212 Since $|L|$ is the height at which the buoyant production of turbulence kinetic energy $(E)$ 213 begins to dominate over shear production, the greater $\left(\overline{w^{\prime} \theta}\right)_{0}$ is (i.e. the smaller $|L|$ is),

214 the bigger $\Delta z_{i}$ and the smaller $z_{s}$ become, because convectively-driven turbulence 215 increasingly dominates over shear-driven turbulence. So, there is a clear relationship 216 between $z_{s}$ and $|L|$ (expressed by (6) in the strongly unstable regime). However, for 217 intermediate unstable regimes the dependence $z_{S}(L)$ is not known.

218 Based on the ABL model described in Sect. 2.1, we define $z_{S}$ as the height where the 219 vertical derivative of $\theta(z)$ reaches a small prescribed fraction of its surface value. Using 
220 this property, in the present model $z_{S}(L)$ is determined by (see details in Appendix 1) 221 evaluating the root of,

$$
\frac{z_{s}}{z_{0 \theta}}=\frac{1}{\alpha_{\psi_{2}}}\left(\frac{z_{0 \theta}}{z_{s}}\right)^{-\alpha_{\psi_{1}}}\left[\frac{1+\gamma_{h}\left(z_{s} /|L|\right)^{\alpha_{1}}}{1+\gamma_{h}\left(z_{0 \theta} /|L|\right)^{\alpha_{1}}}\right]^{-\alpha_{2}},
$$

223 for any value of $L$, assuming that $z_{0 \theta}, \alpha_{1}, \alpha_{2}, \gamma_{h}, \alpha_{\Psi_{1}}$ and $\alpha_{\Psi_{2}}$ are provided. As (14) 224 includes the influence on $z_{S}(L)$ of parameters in both extremes of the stability interval 225 (see Appendix 1), it is expected to provide a good approximation over the whole stability 226 range. As the roughness length for heat, $z_{0 \theta}$, is not provided by $\mathrm{C} 94$, we use here $z_{0}$ 227 instead. Calculations not presented here show that the $z_{S}(L)$ dependences obtained using $228 z_{0 \theta} / z_{S}$ or $z_{0} / z_{S}$ are quite similar (the relation between $z_{0 \theta}$ and $z_{0}$ assumed for this 229 comparison follows Zilitinkevich 1995). Although $z_{0 \theta}$ and $z_{0}$ differ, the proposed method 230 for estimating $z_{s}$ is not very sensitive to the exact value of $z_{0}$ as long as this is small.

$231 u_{*}(L)$, on the other hand, is calculated from

$$
u_{*}\left(z_{s}, L\right)=K_{s}\left[1+\gamma_{m}\left(\frac{z_{s}}{|L|}\right)^{\alpha_{1}}\right]^{\alpha_{2}}\left(\frac{\partial U}{\partial z}\right)_{z_{s}},
$$

233 where, in accordance with the slab model adopted initially (see Sect. 2.1), it is expected 234 that $\partial U / \partial z$ becomes small as $z \rightarrow z_{s}$. Here we assume that in (15) the shear $(\partial U / \partial z)_{z_{s}}$ 235 is constant, and, for convenience, equal to its neutral value. For $|L| \rightarrow \infty$ and at $z=z_{s}$,

236 (1) reduces to $(\partial U / \partial z)_{z_{s n}}=u_{* n} /\left(\kappa z_{s n}\right)$, in accordance with (11), where $z_{s n}$ may be 237 obtained from (12). The validity of the assumption $(\partial U / \partial z)_{z_{s}}=$ const. is tested in 238 Appendix 2.

239 All quantities on the right-hand side of (15) are now known, and hence $u_{*}\left(z_{S}, L\right)$ may be 240 determined in general. Finally, the $U(z)$ profile for the general unstably stratified case, 241 which will be used in the HLR model for calculating $\Delta S(L)$,

$$
U(z)=\frac{u_{*}}{\kappa}\left\{\ln \left(\frac{z}{z_{0}}\right)-3 \ln \left[\frac{1+\sqrt{1+\gamma_{m}(z / L L)^{2 / 3}}}{1+\sqrt{1+\gamma_{m}\left(z_{0} / L\right)^{2 / 3}}}\right]\right\},
$$

243 is obtained by integration of (1), using the velocity gradient expressed by (4) (see W01). 
244 In the above treatment, it was assumed that the synoptic situation does not vary too 245 rapidly compared with the time scales of flow over the ridge. Hence, according to MOST, 246 the effect of $L$ in the surface layer is dominant. As this quasi-steadiness is supported by 247 the C94 campaign, the C94 observations can safely be used for testing the method 248 proposed here. For more unsteady flows, it is likely necessary to use a time-dependent 249 model for the whole ABL, such as that described by Weng and Taylor (2003), for 250 providing upstream profiles $U(z)$ and $\theta(z)$ at different values of $L$. However, this 251 approach would require more input parameters not available in the C94 observations, and 252 their estimation would further increase the empiricism of the proposed method.

253 Summarizing, in this section, assuming that $z_{0}, z_{s f c}, L_{f c}, z_{s n}$ and $u_{* n}$ are known, we 254 propose (14) and (15) for determining $z_{S}(L)$ and $u_{*}(L)$, respectively. $u_{*}(L)$ is then used 255 in the HLR model to calculate $\Delta S(L)$ for flow over orography.

\section{$257 \quad 3$ Results and discussion}

258 The method presented above will be assessed using the observations of C94. These 259 measurements were conducted during the Spring of 1984 and Summer of 1985, over 260 Cooper's Ridge, located to the north-west of Goulburn, in New South Wales, Australia.

261 This is a somewhat isolated north-south oriented, quasi-two-dimensional ridge of uniform

262 low $z_{0}$, located along a valley that forces the air to flow over the hill predominantly from 263 the west side. The windward slope of the ridge (west side) can be well fitted using a 264 simple bell-shaped profile $h(x)=h_{0} /\left\{1+(x / a)^{2}\right\}$ (with $h_{0}=115 \mathrm{~m}$ and $a=400 \mathrm{~m}$ ). 265 The lee side of the ridge falls away to about $0.5 h_{0}$ before rising to another broader ridge.

2673.1 Estimation of parameter values from the data

268 As mentioned in Sect. 2, for determining $z_{S}(L)$ and $u_{*}(L)$, the method developed here 269 requires that $z_{0}, z_{s f c}, u_{* f c}, L_{f c}, z_{s n}$ and $u_{* n}$ be known. From the data collected by C94, 270 we have $u_{* n}=0.35 \mathrm{~m} \mathrm{~s}^{-1}, z_{0}=0.05 \mathrm{~m}$ and $f \approx 9 \times 10^{-5} \mathrm{~s}^{-1}$. Using (13) we thus obtain $z_{\text {in }}$ $271=778 \mathrm{~m}$, and using this value in (12) yields $z_{s n}=39 \mathrm{~m}$.

272 The methodology described in Sect. 2.3 for estimating the flow parameters in the free273 convection regime $\left(z_{s f c}, u_{* f c}, L_{f c}\right.$ and $\left.w_{*}\right)$ is now applied. As $z_{i f c}$ is not supplied by 274 C94, we will use a typical value corresponding to the season and latitude of the region 
275 where the observations were taken. Figures containing the necessary information from 276 ERA-40 Reanalysis, provided by Von Engeln and Teixeira (2013), suggest $z_{i f c}=1550$ $277 \mathrm{~m}$. Next, since $z_{i f c} / z_{0}=3 \times 10^{4}<\sigma=3.45 \times 10^{5}$, we must use (9) to calculate $u_{* f c} / w_{*}=$ 278 0.098. Substituting $u_{* f c} / w_{*}$ and $z_{i f c}$ into (7), we obtain $L_{f c}=-3.6 \mathrm{~m}$, and from (6) we 279 obtain $z_{s f c}=7.2 \mathrm{~m}$. Next, substitution of $z_{i f c}$ in (10) gives $w_{*}=1.74 \mathrm{~m} \mathrm{~s}^{-1}$, which in turn 280 can be used for calculating $u_{* f c}$ from $u_{* f c} / w_{*}=0.098$, yielding $u_{* f c}=0.17 \mathrm{~m} \mathrm{~s}^{-1}$. Table 2811 presents known and estimated parameters of the ABL in the free-convection regime, 282 obtained by the present method and, for comparison, observations from runs $6 \mathrm{~A} 1$ and $2836 \mathrm{~A} 2$ of the field experiment reported by K76, corresponding to a highly convective ABL. 284 As can be seen, the method proposed here seems to predict realistic results.

\begin{tabular}{|l|c|c|c|c|c|c|c|}
\hline Source & $u_{* f c}\left(\mathrm{~m} \mathrm{~s}^{-1}\right)$ & $\left|L_{f c}\right|(\mathrm{m})$ & $z_{s f c}(\mathrm{~m})$ & $z_{i f c}(\mathrm{~m})$ & $z_{s f c} / z_{i f c}$ & $w_{*}\left(\mathrm{~m} \mathrm{~s}^{-1}\right)$ & $u_{* f c} / w_{*}$ \\
\hline $\begin{array}{l}\text { Present } \\
\text { method }\end{array}$ & 0.17 & 3.6 & 7.2 & 1550 & 0.005 & 1.70 & 0.098 \\
\hline Run 6A1 & 0.24 & 5.7 & 10.4 & 2095 & 0.005 & 2.43 & 0.099 \\
\hline Run 6A2 & 0.23 & 6.4 & 12.8 & 2035 & 0.006 & 2.21 & 0.104 \\
\hline
\end{tabular}

Table 1 Parameters of the ABL in the free-convection regime. Line 1: parameters used in the present method. Lines 2-3: similar parameters from runs 6A1 and 6A2 of the experiment described in Kaimal et al.

290 It is interesting that, contrary to what happens in the neutral regime, the ratio $z_{s f c} / z_{\text {ifc }}=$ 2910.005 , estimated above, is significantly lower than the value assumed in (12). This value 292 is of the same order of magnitude as those derived from the measurements of K76, taken 293 in strongly convective conditions (see Table 1). As pointed out before, the smaller $|L|$ is, 294 the more intense the turbulent mixing by large convective eddies in the mixed layer 295 becomes, thereby reducing $z_{s}$. This corroborates, using real data, that the neutral 296 approximation for $z_{s n} / z_{i n}$ cannot be considered realistic over the whole range of 297 variation of $L$, particularly near the free-convection regime.

\subsection{Behaviour of $z_{s}$ as a function of $L$}

300 For a better understanding of the surface-layer structure, it is useful to define a transition

301 height, $z_{t r}$, at which the convective contribution to $U(z)$ is as important as that of the 
neutral log law. Following Kader and Yaglom (1990), from the W01 formulation (4), we

303 can define

$$
Z_{t r}=|L| \gamma_{m}^{-1 / \alpha_{1}} .
$$

305 It is expected that, in moderately to strongly unstable flow regimes $z_{t r}<z_{s}$, i.e. at the top 306 of the surface layer $U(z)$ is no longer logarithmic.

307 Figure 1 presents the variation of $z_{s}$ and $z_{t r}$, with $|L|$, normalized by $z_{s n}$. The solid line 308 represents $z_{S}(L)$, computed using (14). In (14), the coefficients $\alpha_{\Psi_{1}}$ and $\alpha_{\Psi_{2}}$, given by 309 (23), take the values 0.415 and 0.018 , respectively. $z_{t r}(L)$ (dashed line) is computed 310 using (17).

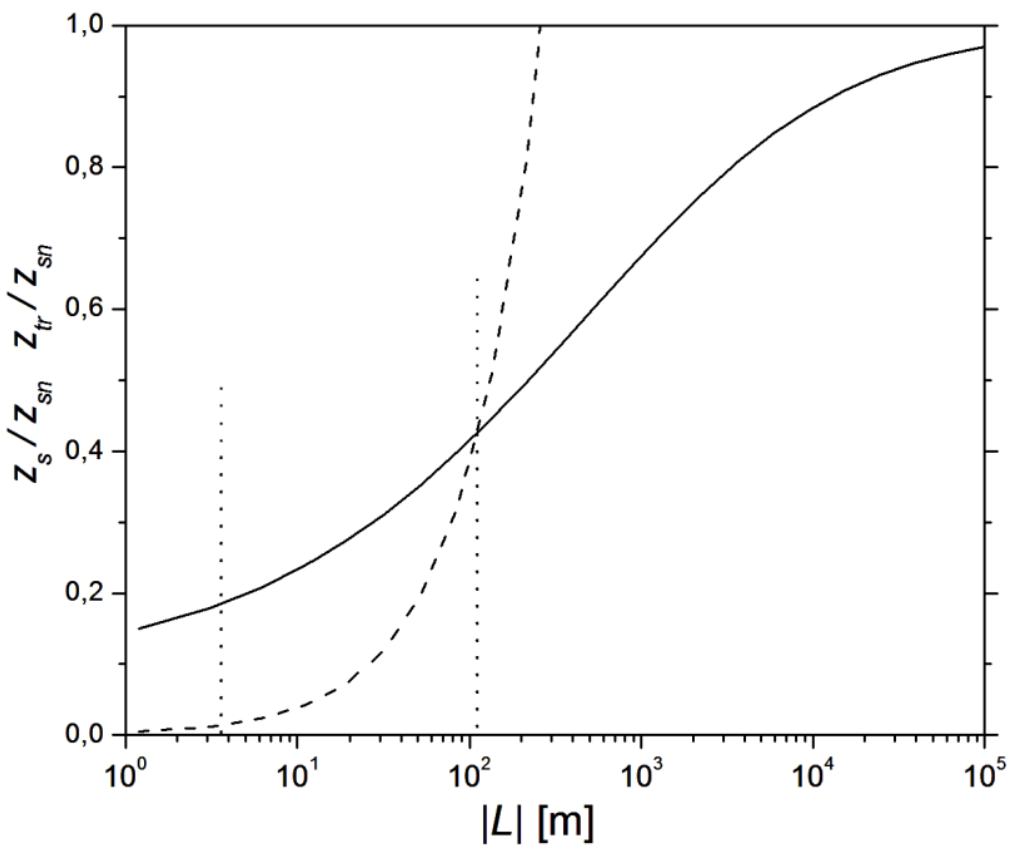

Fig 1 Surface-layer height, $z_{S}$, and transition height, $z_{t r}$, as a function of $|L|$, normalized by the surfacelayer height for a neutral ABL, $z_{s n}$. Solid line: $z_{s}(L)$ obtained from (14), dashed line: $z_{t r}(L)$ obtained from (17). Vertical dotted lines: $\left|L_{f c}\right|=3.6 \mathrm{~m}$ (left), and $\left|L_{t r}\right|=120 \mathrm{~m}$ (right). $z_{s}(L)$ asymptotically approaches the constant values $z_{s n}$ as $|L| \rightarrow \infty$ and $z_{s}(L)=4.5 \mathrm{~m}$ as $|L| \rightarrow 0 . z_{s}\left(L_{f c}\right)=z_{s f c}=7.2 \mathrm{~m}$ (see Table 1 ).

318 The dotted vertical lines correspond to $\left|L_{f c}\right|=3.6 \mathrm{~m}$ (left) as determined previously (see 319 Table 1), and the value of $\left|L_{t r}\right|=120 \mathrm{~m}$ (right) for which $z_{s}(L)=z_{t r}(L)$, i.e. for which 320 the logarithmic and convective contributions to $U(z)$ are equally important. For $|L|>400$ $321 \mathrm{~m}$ the logarithmic portion of $U(z)$ is overwhelmingly dominant compared to the 
322 convective one, and therefore it can be considered that the ABL is in nearly neutral 323 conditions. For $L=L_{f c}$ or lower, the opposite is true, as the flow is nearly in free-

324 convection conditions. $z_{S}(L)$ physically behaves as expected, tending asymptotically to 325 constant values at each extreme of the stability interval $\left(4.5 \mathrm{~m}\right.$ as $|L| \rightarrow 0$, and $z_{s n}$ for

$326|L| \rightarrow \infty)$. Figure 1 illustrates the way in which the surface layer becomes thinner with

327 increasing unstable stratification, because of the progressively higher buoyant production 328 of $E$ in the mixed layer as $|L|$ decreases.

329

$330 \quad 3.3$ Behaviour of $u_{*}$ as a function of $L$

331 Figure 2 presents $u_{*}$ as a function of $|L|$, normalized by $u_{* n}$. The solid line corresponds 332 to $u_{*}(L)$ computed from (15), and the dash-dotted line extends the constant neutral value, $333 u_{* n}=0.35 \mathrm{~m} \mathrm{~s}^{-1}$, over the whole stability interval, for comparison. Figure 2 shows that $334 u_{*}(L)$ decreases with decreasing $|L|$ until it reaches its minimum value $\left(u_{* \min }\right)$ at $L=$ $335\left|L_{\text {min }}\right|$. According to (15), for $|L|<\left|L_{\min }\right|, u_{*}(L)$ would increase monotonically with 336 decreasing $|L|$, in such a way that $u_{*}(L) \rightarrow \infty$ for $|L| \rightarrow 0$. This behaviour occurs 337 because, as $|L| \rightarrow 0$, the term between brackets on the right-hand side of (15) tends to 338 infinity. This is a consequence of the physically unrealistic behaviour of MOST as $|L| \rightarrow$

3390 , producing singularities. For this reason, in Fig. 2 we have assumed that $u_{*}(L)=u_{* \min }$, 340 for $|L| \leq\left|L_{\min }\right|$. 


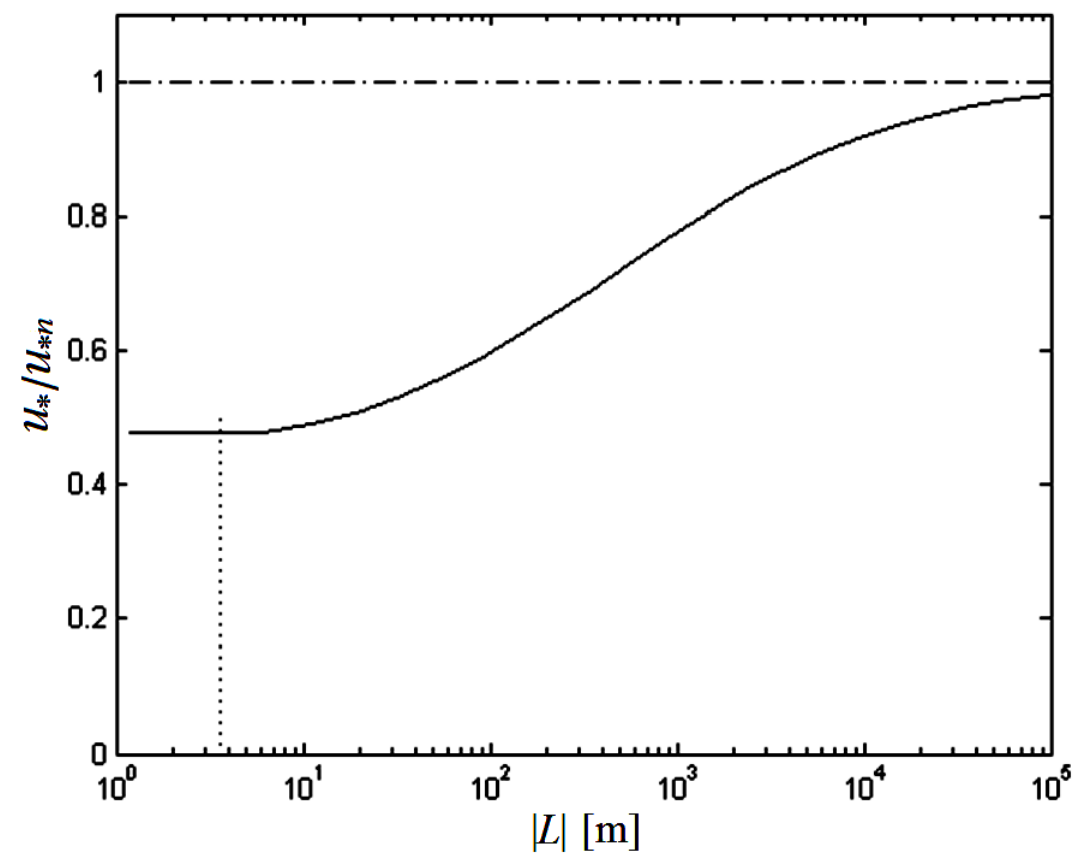

343 Fig 2 Friction velocity $\left(u_{*}\right)$ as a function of $|L|$, obtained from (15) (solid line) and constant $u_{*}$ independent 344 of the stability and equal to its value in the neutral regime $\left(u_{* n}=0.35 \mathrm{~m} \mathrm{~s}^{-1}\right)$ (dash-dotted line). Both 345 quantities are normalized by $u_{* n}$. The vertical dotted line indicates the value of $L$ in the free-convection 346 regime, $\left|L_{f c}\right|=3.6 \mathrm{~m}$ (see Table 1$)$.

348 As can be seen in Fig. 2, $u_{*}(L)$ shows the expected physical behaviour (cf. Fig. 3.7 of 349 Garratt 1992), approaching asymptotically (by design) $u_{* n}$ as $|L| \rightarrow \infty$, and decreasing 350 monotonically with decreasing $|L|$. However, the approach to $u_{* n}$ as $|L| \rightarrow \infty$ is very 351 gradual and $u_{*}$ only takes a value mid-way between the neutral and free-convection limits 352 for an $|L|$ of several hundred metres. Furthermore, the minimum value reached by $u_{*}(L)$ 353 is $u_{* \min }=0.17 \mathrm{~m} \mathrm{~s}^{-1}$ for $L_{\min }=3.4 \mathrm{~m}$. Thus, $u_{* \min }=u_{* f c}$ and $\left|L_{\min }\right|$ almost coincides 354 with $\left|L_{f c}\right|=3.6 \mathrm{~m}$, determined previously (see Table 1). This result further confirms that 355 the assumption of constant $(\partial U / \partial z)_{z_{S}}$ is realistic, and allows obtaining reliable estimates 356 of $u_{*}$ over the whole stability interval.

357 Although $u_{* f c}$, is thus a minimum value of $u_{*}$, it is generally not as low compared with $358 u_{* n}$ as might be expected. The case under consideration here, where $u_{* f c} / u_{* n} \approx 0.5$, 359 which is not particularly low (see Sect. 3.1, Table 1), is a good example. This result 360 ultimately suggests that a purely-thermal regime is unlikely (it was not realized in the 361 C94 measurements, in particular). For these reasons, under nearly free-convection 
362 conditions both $u_{* f c}$ and $L_{f c}$ differ substantially from zero, as is confirmed by the

363 observations of K76 (see Table 2), and further corroborated for a very unstable surface-

364 layer case by Steeneveld et al. (2005). This is what allows MOST to be used here for

365 describing a highly convective ABL.

366

367 3.4 Flow speed-up calculation

368 Since calculating $\Delta S(L)$ using the HLR model requires knowing $u_{*}(L)$, the main purpose

369 of this section is to use the behaviour of $\Delta S(L)$ predicted by that model to indirectly

370 assess the dependence on stability of $u_{*}(L)$ (and also of $z_{S}(L)$ ) established in the method

371 proposed here, by comparison with values of $\Delta S$ measured over a wide range of $L$ by

372 C94, and simulated numerically using the FLEX model.

373 Suppose that at a hilly location $\Delta S(L)$ needs to be estimated, assuming that the only

374 available parameters are $z_{0}$ and the mean wind speed, $U(z)$, measured at a suitably low

375 height such that, according to MOST, (11) is approximately valid for any $L$. Equation 11

376 can then be used for estimating $u_{* n}$. Once $z_{0}$ and $u_{* n}$ are known, the present method

377 allows systematically obtaining $z_{S}(L)$, then $u_{*}(L)$ and finally $\Delta S(L)$, for the whole

378 unstable stratification parameter range.

379 In the specific case under consideration here, first using as input parameters $u_{* n}=0.35 \mathrm{~m}$

$380 \mathrm{~s}^{-1}$ and $z_{0}=0.05 \mathrm{~m}$ (from C94), $u_{*}(L)$ is calculated using the proposed method. Next, this

$381 u_{*}(L)$ is used in the HLR model applied to flow over Cooper's ridge to calculate $\Delta S(L)$.

$382 \Delta S(L)$ is also calculated assuming that $u_{*}=$ const. $=u_{* n}$, regardless of the observed $L$.

383 This simpler choice, often used for estimating $\Delta S(L)$ in flow over orography (e.g. W97),

384 is what the present approach aims to improve. Finally, the $\Delta S$ values are compared, for a

385 range of $L$, between the HLR model, the C94 measurements, and the FLEX model.

386 For the sake of simplicity the HLR and FLEX models are not described in detail here. A

387 brief description of the HLR model can be found in W97 or A09. FLEX is a microscale388 mesoscale, nonlinear and non-hydrostatic model, which was developed and validated 389 against experimental and field data by Argaín (2003) and A09. This model has been

390 tested and used extensively, namely by Teixeira et al. (2012, 2013a, 2013b) for assessing 391 analytical mountain wave drag predictions in 2D flows by comparison with numerical 392 simulations. 
393 All the numerical simulations presented here used a main grid of $(160 \times 364)$ points for a 394 domain of $(8000 \times 2000) \mathrm{m}$ size. The horizontal domain extent is $20 a(7 a$ upstream of 395 the ridge maximum and $13 a$ downstream). From $z=40 \mathrm{~m}$ downward the level of grid 396 refinement is gradually increased, and the lowest level is at a similar distance to the 397 surface as the observations $(\approx 0.15 \mathrm{~m})$. At the surface a no-slip condition is used, and $398\left(\overline{w^{\prime} \theta}\right)_{0}$ and other turbulent quantities (turbulent kinetic energy, $E$, and dissipation $\varepsilon$ of $399 E$ ), are specified, for each $L$, by assuming that viscous dissipation balances shear and 400 buoyancy production. At the upper boundary, constant $U$ and $\theta$ are prescribed, and $L$ and 401 the derivatives of $E$ and $\varepsilon$ are set to zero.

402 Observations, and both theoretical and numerical predictions of $\Delta S$ as a function of $|L|$ 403 are shown in Fig. 3, at $z=8 \mathrm{~m}$ and $z=16 \mathrm{~m}$. The HLR model is applied in two cases: a) $404 u_{*}=u_{* n}$, regardless of $|L|$ (dashed line), and $\mathrm{b}$ ) the friction velocity is calculated for each $405|L|$, using the method proposed here (15) (solid line).

406

407

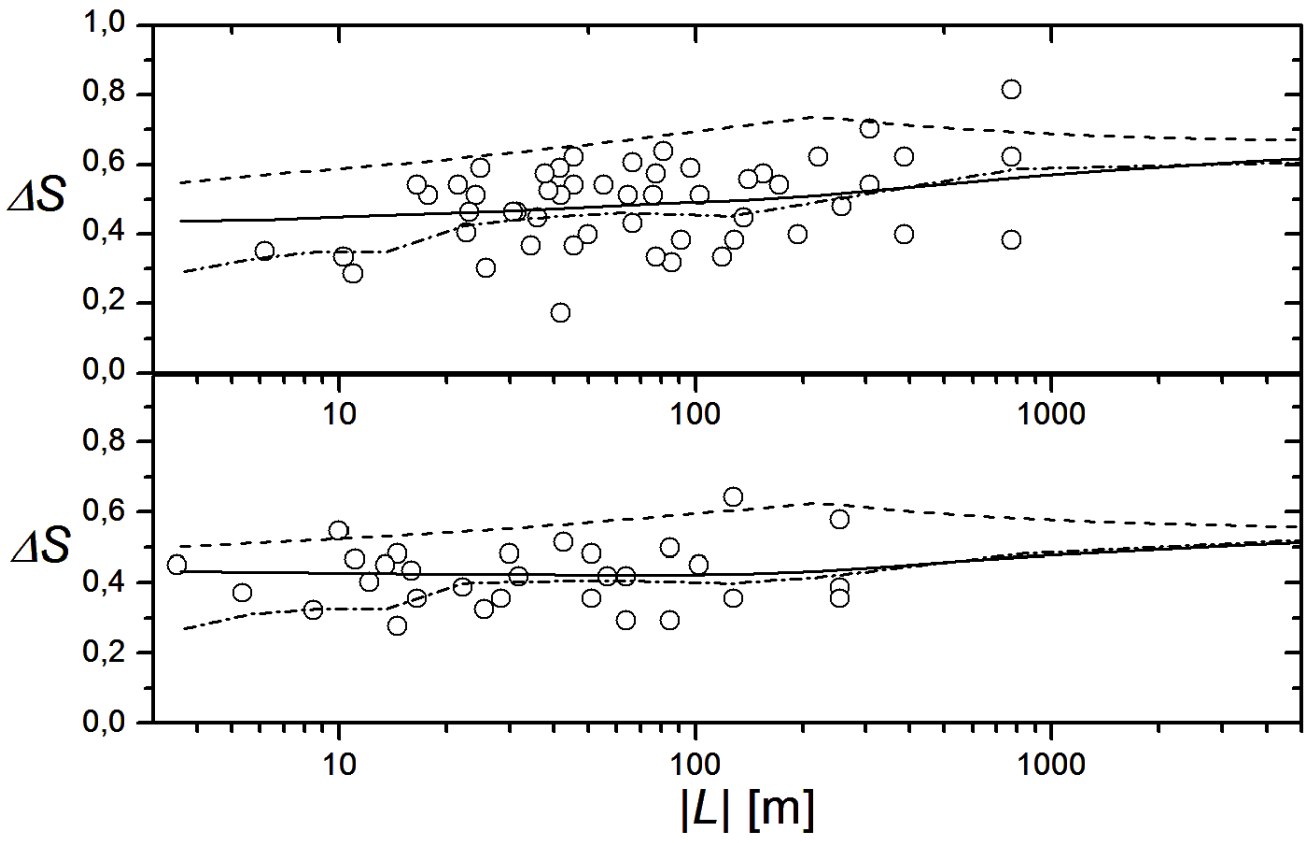

Fig 3 Variation of the fractional speed-up $(\Delta S)$ as a function of stability, above the hill crest, at the heights $z=8 \mathrm{~m}$ (top) and $z=16 \mathrm{~m}$ (bottom). Solid line: $u_{*}$ computed using (15); dotted line: $u_{*}$ kept constant, regardless of the stability, and equal to the neutral ABL value $\left(u_{* n}=0.35 \mathrm{~m} \mathrm{~s}^{-1}\right)$; dash-dotted line: FLEX model; symbols: observations from C94. 
413 The significant differences between the $\Delta S$ curves, obtained using the two different 414 definitions of $u_{*}$, reveals that $\Delta S$ is very sensitive to the dependence of $u_{*}$ on $|L|$, as 415 shown by A09 for the stable case. The results assuming $u_{*}=u_{* n}$ (dashed lines) 416 overestimate the observations considerably. In both panels of Fig. 3, the improvement in 417 the performance of the theoretical model, owing to the new method of calculating $u_{*}$ 418 (solid lines), is significant over the whole stability interval. In general, this new method 419 produces results much closer to both the field measurements (despite the considerable 420 scatter in the data) and the numerical simulation results. $\Delta S$ calculated from the 421 theoretical model with $u_{*}$ depending on $L$ has a rather flat variation with $|L|$, especially at $422 z=16 \mathrm{~m}$, and although decreasing more substantially with $|L|$ at $z=8 \mathrm{~m}$, slightly 423 overestimates both the measurements and the numerical simulations for the lowest values 424 of $|L|$.

425

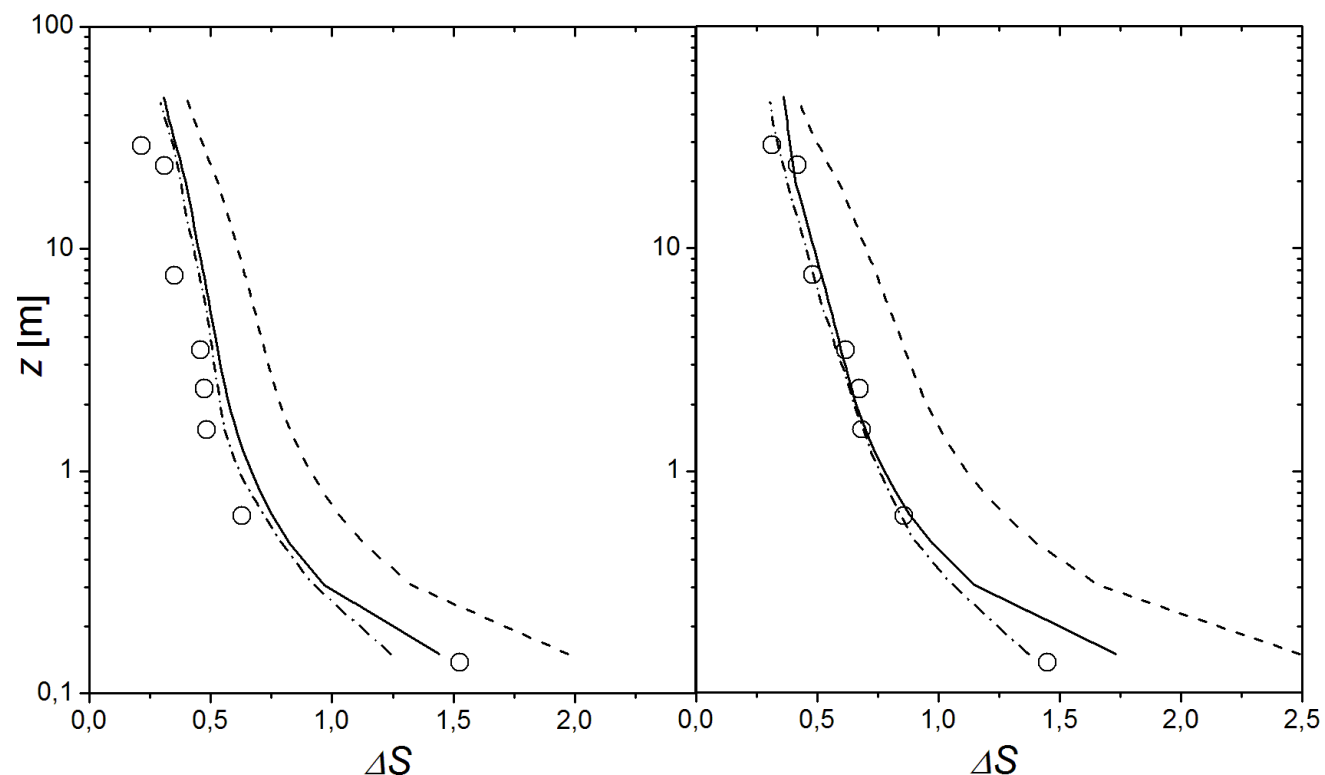

427 Fig 4 Profiles of the fractional speed-up ratio $(\Delta S)$ above the hill crest, for $|L|=33 \mathrm{~m}($ left) and $|L|=222 \mathrm{~m}$ 428 (right). Solid line: $u_{*}$ computed using (15); dashed line: $u_{*}$ kept constant at $u_{* n}=0.35 \mathrm{~m} \mathrm{~s}{ }^{-1}$; dash-dotted 429 line: FLEX model; symbols: observations from C94.

431 Profiles of observations (C94), and both theoretical and numerical predictions of $\Delta S$ 432 directly above the hill crest, for $|L|=33 \mathrm{~m}$ (left panel) and $|L|=222 \mathrm{~m}$ (right panel) are 433 shown in Fig. $4 .|L|=33 \mathrm{~m}$ and $|L|=222 \mathrm{~m}$ correspond to strong and moderately weak 
434 unstable stratification, respectively. In both cases, the proposed method compares better

435 both with the numerical model and with the field data, although it slightly overestimates

436 the observations in the more unstable case. Nevertheless, a general decrease of $\Delta S$ as one

437 shifts from the higher to the lower $|L|$ value is qualitatively reproduced. Given the

438 precision of the measurements and flow assumptions, not too much importance should be

439 attached to this overestimate, which also occurs in the numerical simulations

440 (consistently, a similar discrepancy can be detected for the theoretical model on the far

441 left of Fig. 3 at $z=8 \mathrm{~m}$ ).

$442 \Delta S$ is much more severely overestimated, in both cases, by the profiles with a prescribed

443 constant $u_{*}=u_{* n}$, due essentially to the significant fractional deviation between $u_{* n}$ and

444 the more accurate value of $u_{*}$ determined from (15). This fractional deviation amounts to

$445 \sim 45 \%$ for $|L|=33 \mathrm{~m}$ and to $\sim 35 \%$ for $|L|=222 \mathrm{~m}$ (see Fig. 2), but this does not translate

446 into proportional deviations for $\Delta S$, as the value of $\Delta S$, where $u_{*}$ is calculated from (15),

447 actually becomes closer to that where $u_{*}=u_{* n}$ as $|L|$ decreases (see Fig. 3). The fact that

448 there is such a large difference in the results using $u_{*}(L)$ and $u_{*}=u_{* n}$ for the weakly

449 unstable case might seem suspect, but Fig. 2 explains it, since for $|L|=222 \mathrm{~m}, u_{*}(L)$ still

450 differs very substantially from $u_{* n}$.

451 It should be pointed out that at the lowest measurement level, $\Delta S$ should depend very 452 weakly on $L$, because near enough to the ground the flow is always approximately 453 neutral. The overestimate of the measured $\Delta S$ at that level by the theoretical model for $|L|$

$454=222 \mathrm{~m}$ can probably be attributed to an inherent bias of the HLR solution, noted by 455 W97 and A09.

4574 Summary and conclusions

458 In this paper, we propose a new method for estimating two scaling parameters of the 459 ABL: the surface-layer height $z_{s}$ and the friction velocity $u_{*}$, as a function of stability 460 (quantified by the Obukhov length scale $L$ ), for an unstable ABL. These two parameters 461 are important for characterizing the unstable $\mathrm{ABL}$, in particular its coupling with the 462 overlying convective mixed layer. Moreover, a correct estimation of $u_{*}$, whose 463 dependence on $L$ is often not accounted for in a physically consistent way, is crucial for 
464 producing accurate predictions of the speed-up $(\Delta S)$ in flow over hills, which is relevant 465 for a number of engineering applications.

466 Using a physical approach that is developed specifically for unstable conditions, via a 467 combination of MOST and convective mixed-layer scaling, our model takes into account 468 the fact that $z_{s}$ decreases as the unstable stratification becomes stronger, due to erosion of 469 the surface-layer eddies by more energetic buoyancy-dominated eddies from the 470 convective mixed layer. The model also takes into account the fact that $u_{*}$ decreases as 471 the ABL becomes more unstable, attaining a minimum value, but does not, in general, 472 approach zero in the free-convection limit, unless the wind vanishes completely (in which 473 case the concept of $\Delta S$ loses its meaning). The variation of $u_{*}$ affects the turbulent fluxes 474 of various properties, and consequently the mean profiles of those properties, including 475 the wind speed $U(z)$, which determines the behaviour of $\Delta S$.

476 Procedures to obtain boundary-layer parameters in the neutral and free-convection 477 regimes, and for bridging across these regimes, to cover the complete unstable ABL 478 parameter range, were developed and tested using available field data. The performance 479 of the model was then evaluated more comprehensively, by comparing predictions of $\Delta S$ 480 in unstable conditions, using the linear model of HLR incorporating the new friction 481 velocity formulation, against measurements from C94, and numerical simulations of the 482 FLEX mesoscale-microscale model. Agreement was found to be substantially improved 483 relative to results where $u_{*}$ is held constant. This emphasizes the importance of 484 accounting for the full dynamics of the unstable ABL, including the variation of $u_{*}$ and $z_{S}$ 485 with stability, for correctly estimating $\Delta S$. The proposed method, whose possible 486 applications are not limited to improving the calculation of $\Delta S$, should be seen as a 487 preliminary step in the development of better tools for the parametrization of unstable 488 ABLs. Further validation of this method by comparison with observations remains 489 necessary.

491 Acknowledgements The authors thank two anonymous referees and the co-editors for insightful 492 comments, which substantially improved this article. M.A.C.T. acknowledges the financial support of the 493 European Commission, under Marie Curie Career Integration Grant GLIMFLO, contract PCIG13-GA494 2013-618016. P.M.A.M. acknowledges the financial support of FCT, under Grant RECI/GEO495 MET/0380/2012. 


\section{Appendix 1. Accuracy of the $(\partial \theta / \partial z)_{z_{s}}=$ const. approximation}

498 In the present model, the form of $z_{S}(L)$ is established using the temperature gradient $499 \partial \theta / \partial z$, which can be obtained from (2) and (4), yielding

500

$$
\frac{\partial \theta}{\partial z}=\theta_{*}^{\prime}\left[1+\gamma_{h}\left(\frac{z}{|L|}\right)^{\alpha_{1}}\right]^{-\alpha_{2}} z^{-1}
$$

501 where $\theta_{*}{ }^{\prime}=P r_{t} \theta_{*} / \kappa$. According to (18), $\partial \theta / \partial z \rightarrow 0$ as $z \rightarrow \infty$. This is consistent with

502 the assumption that $\theta=$ const. in the mixed layer, so, at $z=z_{s}$ the derivative $(\partial \theta / \partial z)_{z_{s}}$

503 should be suitably small, and this smallness is exploited to obtain $z_{S}$. Note that a similar

504 condition could be based on the mean velocity gradient (1), but we think that $\theta=$ const.

505 is more reliable in the mixed layer, since $U(z)$ profiles may exhibit non-negligible shear

506 above the surface layer, due to variation of the pressure perturbation induced by the

507 orography with height or the Coriolis force. Taking this into account, the ratio

508

$$
\Psi\left(z_{s}, L\right)=\frac{(\partial \theta / \partial z)_{z_{s}}}{(\partial \theta / \partial z)_{z_{0 \theta}}}=\left[\frac{1+\gamma_{h}\left(z_{s} / L \mid\right)^{\alpha_{1}}}{1+\gamma_{h}\left(z_{0 \theta} / L\right)^{\alpha_{1}}}\right]^{-\alpha_{2}} \frac{z_{0 \theta}}{z_{s}}
$$

509 implicitly determines $z_{S}(L)$, if the form of the function $\Psi\left(z_{S}, L\right)$ is known. In (19)

$510(\partial \theta / \partial z)_{z_{S}}$ and $(\partial \theta / \partial z)_{z_{0 \theta}}$ are obtained by evaluating (18) at $z_{S}$ and the temperature

511 roughness height, $z_{0} \theta$, respectively. As defined by (19), $\Psi\left(z_{s}, L\right)$ varies monotonically

512 from 1 to zero as $z_{0 \theta} / z_{S}$ decreases. Moreover, $\Psi\left(z_{S}, L\right)$ depends only weakly on $L$ : by

513 substituting $L_{f c}, z_{s f c}, z_{s n}$ and $L_{n}=\infty$ (see Sect. 3.1, Table 1) into (19) we may calculate

514 the ratio $\Gamma=\Psi\left(z_{s n}, L \rightarrow-\infty\right) / \Psi\left(z_{s f c}, L_{f c}\right) \approx 0.6$, which is of order 1 .

515 The limits of $\Psi\left(z_{S}, L\right)$ at the theoretical extremes of the stability interval are, respectively,

$$
\Psi_{s f c}=\Psi\left(z_{s f c}, L \rightarrow 0\right)=\lim _{|L| \rightarrow 0} \Psi(L)=\left(\frac{z_{0 \theta}}{z_{s f c}}\right)^{\alpha_{1} \alpha_{2}+1},
$$

$$
\Psi_{s n}=\Psi\left(z_{s n}, L \rightarrow \infty\right)=\lim _{|L| \rightarrow \infty} \Psi(L)=\frac{z_{0 \theta}}{Z_{s n}}
$$

518 For both strongly and weakly unstable flows, (20) - (21) suggest that $\psi\left(z_{s}, L\right) \propto\left(z_{0 \theta} /\right.$

$\left.519 z_{s}\right)^{\alpha}$, where $\alpha$ is a dimensionless constant. Taking this result into account, we 
520 hypothesize that this form holds for the whole stability interval, yielding the following 521 approximate definition for $\psi\left(z_{s}, L\right)$,

522

$$
\Psi\left(z_{s}, L\right)=\alpha_{\psi_{2}}\left(\frac{z_{0 \theta}}{z_{s}}\right)^{\alpha_{\psi_{1}}},
$$

523 where $\alpha_{\Psi_{1}}$ and $\alpha_{\Psi_{2}}$ are dimensionless constants. These two constants can be determined

524 by taking the limits of (22) in the free-convection and neutral regimes, and comparing the 525 corresponding expressions with (20) and (21), respectively. This produces a set of two 526 equations, which may be solved for $\alpha_{\Psi_{1}}$ and $\alpha_{{ }_{Y}}$, yielding

$$
\alpha_{\psi_{1}}=\ln \left[\frac{\Psi\left(z_{s n}, L \rightarrow \infty\right)}{\Psi\left(z_{s f c}, L_{f c}\right)}\right] / \ln \left(\frac{z_{s f c}}{z_{s n}}\right) \quad \alpha_{\psi_{2}}=\Psi\left(z_{s f c}, L_{f c}\right)\left(\frac{z_{0 \theta}}{z_{s f c}}\right)^{-\alpha_{\psi_{1}}}
$$

528 By combining (19) and (22), (14) is obtained.

530 Appendix 2. Accuracy of the $(\partial U / \partial z)_{z_{s}}=$ const . approximation

531 Here we show that the approximation $(\partial U / \partial z)_{z_{s}}=$ const., used in Sect. 2.4 for

532 evaluating $u_{*}$, is supported by measurements. Let us consider the following ratio, by 533 using MOST,

$$
\beta_{\text {MOST }}=\frac{(\partial U / \partial z)_{z_{s n}}}{(\partial U / \partial z)_{z_{s c}}}=\underbrace{\left(\frac{c_{f c} \beta_{1}|f|}{c_{S L} c_{\text {zin }}}\right)}_{\alpha_{5}}) \frac{L_{f c} \mid}{u_{* f c}},
$$

535 where $\beta_{1}=\left(1+\gamma_{m} c_{f c}^{\alpha_{1}}\right)^{\alpha_{2}}$. Equation 24 is obtained by combining (1), (4), (6), (12) and 536 (13). Using parameters from C94 (see Sect. 3.1) we obtain $\alpha_{5}=0.0466 \mathrm{~s}^{-1}$. For the values 537 of $L_{f c}$ and $u_{* f c}$ shown in Table 1 , this yields $\beta_{M O S T}=0.99$. The remarkable closeness of 538 this value to 1 is fortuitous, although it obviously depends on the values adopted for $c_{z i n}$, $539 c_{f c}$ and $c_{S L}$. For checking further the approximation $\beta_{M O S T} \approx 1$ we use the K76 540 observations (keeping the same $\alpha_{5}$ ), which were carried out in a daytime well-mixed 541 CBL, with evidence of significant heat and momentum entrainment through the capping 542 inversion.

543

\begin{tabular}{|c|c|c|c|c|c|c|}
\hline Run & $u_{* f c}\left(\mathrm{~m} \mathrm{~s}^{-1}\right)$ & $\left|L_{f c}\right|(\mathrm{m})$ & $z_{i f c}(\mathrm{~m})$ & $w_{*}\left(\mathrm{~m} \mathrm{~s}^{-1}\right)$ & $w_{*} / z_{i f c}\left(\mathrm{~s}^{-1}\right)$ & $\beta_{M O S T}$ \\
\hline 6A1 & 0.24 & 5.7 & 2095 & 2.43 & $1.16 \times 10^{-3}$ & 1.1 \\
\hline
\end{tabular}


\begin{tabular}{c|c|c|c|c|c|c|c|}
\hline $6 \mathrm{~A} 2$ & 0.23 & 6.4 & 2035 & 2.21 & $1.09 \times 10^{-3}$ & 1.3 \\
Table 2 CBL parameters measured by Kaimal et al. (1976). Columns 6 and 7 show, respectively, $w_{*} / z_{\text {ifc }}$,
\end{tabular}

545 and $\beta_{\text {MOST }}$, calculated from the data (the second quantity by using (24)).

547 Table 2 shows CBL parameters obtained by K76, corresponding to the runs with the 548 smallest values of $|L|$, typical of nearly free-convection regimes. As can be seen, the 549 values of $\beta_{\text {MOST }}$ are close to 1 , corroborating the hypothesis $\beta_{\text {MOST }} \approx 1$. Moreover, 550 column 6 supports (10), proposed by Venkatram (1978), since $c_{5}=w_{*} / z_{i f c}$ varies within

551 a narrow range. Venkatram (1978) estimated $c_{5}=1.12 \times 10^{-3} \mathrm{~s}^{-1}$, which is quite close to 552 both values shown in Table 2. Therefore, the assumption that $c_{5}$ is a constant is plausible.

553 If the assumption $\beta_{\text {MOST }}=1$ is accepted, (24) defines a relationship between $\alpha_{5},\left|L_{f c}\right|$ and $554 u_{* f c}$. If parameters $c_{f c}$ and $c_{z i n}$ are assumed to be non-adjustable, this is equivalent to a 555 relation between $\left|L_{f c}\right|, u_{* f c}$ and $c_{S L}$. Equation 13 is only applicable if $|L| \rightarrow \infty$ (a rarely 556 observed situation), so the parameter $c_{z i n}$ may have a considerable uncertainty. 557 Zilitinkevich et al. (2012) and Garratt (1992) discuss this topic at length. It would be 558 interesting to explore the constraint defined by (24) further to develop relations other than 559 (13) for estimating $z_{i n}$, but that is beyond the scope of the present paper.

560 We also carried out a similar analysis using the classical free-convection formulation of 561 Prandtl (1932) for the mean velocity gradient,

$$
\left(\frac{\partial U}{\partial z}\right)_{z_{s f c}}=\frac{c_{u} u_{* f c}\left(\kappa\left|L_{f c}\right|\right)^{1 / 3}}{z_{s f c}^{4 / 3}}=\frac{c_{u} \kappa^{1 / 3}}{c_{f c}^{4 / 3}} \frac{u_{* f c}}{\left|L_{f c}\right|},
$$

563 where $c_{u}=1.7$. In this case we would obtain a ratio $(\partial U / \partial z)_{z_{s f c}} /(\partial U / \partial z)_{z_{s n}}$ with a 564 similar parameter dependence as (24) and $\alpha_{5}=0.0453 \mathrm{~s}^{-1}$. The proximity between the 565 values of $\alpha_{5}$ obtained using both formulations for $(\partial U / \partial z)_{z_{s f c}}$ confirms that the MOST

566 formulation adopted here is physically consistent, hence it may be used to describe the 567 free-convection regime.

\section{References}

570 Argaín JL (2003) Modelação de Escoamentos Atmosféricos: Efeitos Orográficos e de Camada Limite (in 571 Portuguese). Ph.D. Thesis, Universidade do Algarve, 275 pp 
Argaín JL, Miranda PMA, Teixeira MAC (2009) Estimation of the friction velocity in stably stratified boundary-layer flows over hills. Boundary-Layer Meteorol 130: 15-28 stability dependence: The mean field. Boundary-Layer Meteorol 69: 173-199 J Atmos Sci 27: 1211-1213

Garratt JR (1992) The Atmospheric Boundary Layer, Cambridge University Press, 316 pp

Hunt JCR, Leibovich SL, Richards KJ (1988) Turbulent Shear Flows Over Low Hills. Q J R Meteorol Soc 29: $16-26$

Kader BA, Yaglom AM (1990), Mean Fields and Fluctuation Moments in Unstably Stratified Turbulent Boundary Layers, J Fluid Mech 212: 637-662

Kaimal JC, Wyngaard JC, Haugen DA, Cote OR, Izumi Y, Caughey SJ, Readings CJ (1976) Turbulence Structure in the Convective Boundary Layer. J Atmos Sci 33: 2152-2169

McNaughton KG (2004) Turbulence Structure of the Unstable Atmospheric Surface Layer and Transition to the Outer Layer. Boundary-Layer Meteorol 112: 199-221

588 Prandtl L (1932) Meteorologische Anvendungen der Str“omungslehre. Beitr Phys Fr Atmos 19: 188-202

Rossby CG, Montgomery RB (1935) The layers of frictional influence in wind and ocean currents. Pap Phys Oceanogr Meteorol 3: $101 \mathrm{pp}$

Schumann U (1988) Minimum Friction Velocity and Heat Transfer in the Rough Surface Layer of a Convective Boundary Layer. Boundary-Layer Meteorol 44: 311-326

Steeneveld GJ, Holtslag AAM, DeBruin HAR (2005) Fluxes and gradients in the convective surface layer and the possible role of boundary-layer depth and entrainment flux, Boundary-Layer Meteorol 116: $237-252$

Stull, RB (1988) An Introduction to Boundary-Layer Meteorology, Kluwer Academic Publishers, Dordrecht,, $670 \mathrm{pp}$

Stull, RB (2011) Meteorology for Scientists and Engineers, $3^{\text {rd }}$ edition, University of British Columbia, Vancouver British Columbia, 938 pp

Sykes RI, Henn DS, Lewellen WS (1993) Surface-layer description under free-convection conditions. Q J

602 R Meteorol Soc 119: 409-421

Teixeira MAC, Argaín JL, Miranda PMA (2012) The importance of friction in mountain wave drag amplification by Scorer parameter resonance. Q J R Meteorol Soc 138: 1325-1337

Teixeira MAC, Argaín JL, Miranda PMA (2013a) Drag produced by trapped lee waves and propagating mountain waves in a two-layer atmosphere. Q J R Meteorol Soc 139: 964-981

Teixeira MAC, Argaín JL, Miranda PMA (2013b) Orographic drag associated with lee waves trapped at an inversion. J Atmos Sci 70: 2930-2947 
608 Venkatram A (1978) Estimating the convective velocity scale for diffusion applications. Boundary-Layer

609 Meteorol 15: 447-452

610 Von Engeln A and Teixeira J (2013) A planetary boundary-layer height climatology derived from ECMWF

611 re-analysis data. J. Clim 26: 6575-6590

612 Weng W (1997) Stably stratified boundary-layer flow over low hills: A comparison of model results and

613 field data. Boundary-Layer Meteorol 85: 223-241

614 Weng W, Taylor PA (2003) On modelling the one-dimensional atmospheric boundary layer. Boundary-

615 Layer Meteorol., 107:371-400

616 Wilson DK (2001) An Alternative Function for the Wind and Temperature Gradients in Unstable Surface

617 Layers. Boundary-Layer Meteorol 99: 151-158

618 Zilitinkevich SS (1995) Non-local turbulent transport: Pollution dispersion aspects of coherent structure of

619 convective flows. Air Pollution Theory and Simulation. Power H, Moussiopoulos N, Brebbia CA, Eds.,

620 Vol. I, Air Pollution III, Computational Mechanics Publications, 53-60.

621 Zilitinkevich SS, Hunt JCR, Grachev AA, Esau IN, Lalas DP, Akylas E, Tombrou M, Fairall CW,

622 Fernando HJS, Baklanov AA, Joffre SM (2006) The influence of large convective eddies on the

623 surface-layer turbulence. Q J R Meteorol Soc 132: 1423-1456

624 Zilitinkevich SS, Tyuryakov SA, Troitskaya YI, Mareev EA (2012) Theoretical Models of the Height of 625 the Atmospheric Boundary Layer and Turbulent Entrainment at Its Upper Boundary. Izvestiya,

626 Atmospheric and Oceanic Physics 48(1): 133-142 